\title{
Philosophiques
}

\section{Pour une épistémologie du concept d'association chez Émile Durkheim et chez Max Weber}

\section{Olivier Perru}

Volume 27, numéro 2, automne 2000

URI : https://id.erudit.org/iderudit/004966ar

DOI : https://doi.org/10.7202/004966ar

Aller au sommaire du numéro

Éditeur(s)

Société de philosophie du Québec

ISSN

0316-2923 (imprimé)

1492-1391 (numérique)

Découvrir la revue

Citer cet article

Perru, O. (2000). Pour une épistémologie du concept d'association chez Émile Durkheim et chez Max Weber. Philosophiques, 27(2), 351-376.

https://doi.org/10.7202/004966ar
Résumé de l'article

Le concept d'association chez Durkheim se rapporte d'abord à la société vue comme réalité autonome et individualisé. Le développement de l'association sociale est mesurable par la division du travail qui constitue la solidarité sociale. Le texte discute de la validité de ce regard sur la société comme unité de vie et du fondement biologique de cette unité. Les recherches des zoologistes du XIXe siècle sur l'unification progressive du vivant permettent à Durkheim de proposer une vision organique de l'État, centre et synthèse de l'organisation. Ce fondement biologique reste cohérent avec les spécificités humaines des faits sociaux. Le point de départ de Max Weber est tout autre : c'est l'individu en tant que sujet libre d'activité et capable de coopération. Les diverses formes de coopération sont alors au fondement des structures sociales qu'analyse Weber à travers l'histoire. Les deux méthodes peuvent être comparées dans leur capacité à rendre compte de la réalité des structures associatives dans la société. 


\section{Pour une épistémologie du concept d'association chez Émile Durkheim et chez Max Weber}

OLIVIER PERRU

Institut Supérieur agricole de Beauvais

olivier.perru@isab.fr

RÉSUMÉ. - Le concept d'association chez Durkheim se rapporte d'abord à la société vue comme réalité autonome et individualisé. Le développement de l'association sociale est mesurable par la division du travail qui constitue la solidarité sociale. Le texte discute de la validité de ce regard sur la société comme unité de vie et du fondement biologique de cette unité. Les recherches des zoologistes du XIX $\mathrm{X}^{\mathrm{e}}$ siècle sur l'unification progressive du vivant permettent à Durkheim de proposer une vision organique de l'État, centre et synthèse de l'organisation. Ce fondement biologique reste cohérent avec les spécificités humaines des faits sociaux. Le point de départ de Max Weber est tout autre : c'est l'individu en tant que sujet libre d'activité et capable de coopération. Les diverses formes de coopération sont alors au fondement des structures sociales qu'analyse Weber à travers l'histoire. Les deux méthodes peuvent être comparées dans leur capacité à rendre compte de la réalité des structures associatives dans la société.

ABSTRACT. - In Durkheim's works, the concept of association firstly relates to society, considered as an autonomous reality. The development of this social association can be measured by the division of labour, which builds up social solidarity. This paper argues about this view of society as a unit of life and its biological foundations. Concerning the progressive unification in living beings, researches of french zoologists during the $19^{\text {th }}$ century enable Durkheim to give a new organicist conception of state. However, this biological founding remains consistent with the human specificities of social facts. Max Weber's starting point is entirely different because he deals with individual as a free subject, able to cooperate. So, diverse ways to cooperate are the foundings of social structures analyzed by Weber.

\section{Introduction}

Faut-il chercher un fondement philosophique dans l'approche du concept d'association chez Durkheim et chez Weber ? À première vue, cela semble paradoxal. En effet, les grands précurseurs et initiateurs de la sociologie ont voulu garantir l'autonomie de leur science du point de vue de sa méthode et de son objet. Toutefois, la discipline qu'ils fondent est, à leur époque, parmi les dernières nées. Ils ne peuvent donc se passer des autres branches du savoir humain, et ce, à des titres divers. Déjà, Saint-Simon empruntait à la biologie de Lamarck et de Geoffroy Saint-Hilaire le concept d'organisme, d'une part, la méthode expérimentale, d'autre part. De même Durkheim empruntera à la biologie de son temps certaines approches de l'organisme vivant dont il tentera 
de tirer des analogies valables dans le domaine social. Il emploiera le concept de division du travail dans l'analyse du progrès des sociétés. Il l'empruntera non seulement à l'économie du début du XIX ${ }^{\mathrm{e}}$ siècle, mais surtout aux spéculations des biologistes de son temps; ceux-ci cherchaient en effet à rendre compte de la différenciation des organes dans les organismes supérieurs par une tendance à la complexification sous la motion de la division du travail. Quant à Weber, il s'appuie beaucoup sur une critique du comportement intellectuel et moral de l'individu, une critique de ses connaissances et de son agir ; on pourrait presque dire qu'au fondement des analyses de Weber, on trouve une épistémologie, même si celle-ci ne fait que naître de son temps.

Dans une étude de la signification du concept d'association chez Durkheim et Weber, comment pourrait-on exclure l'héritage de la philosophie politique, alors que celle-ci a beaucoup plus de rapport avec des concepts relatifs aux sociétés humaines que la biologie ou l'économie. Même si la méthode et l'objet propres à la sociologie sont autonomes, le sociologue ne peut pas répudier pour autant tout lien entre sa discipline et les questions que se pose inévitablement le philosophe politique. Par ailleurs, des concepts comme "association » et " coopération » relèvent d'une expérience spécifique qui fonde justement la dimension politique de l'homme, et qui n'est pas sans interagir avec sa dimension morale. Même si, chez Durkheim, le fait social est considéré pour lui-même dans son objectivité, comment rejeter à la fois l'expérience humaine de coopération qui le fonde et la signification philosophique qui en dérive ? Ainsi, chez Durkheim comme chez Weber, les conclusions relatives aux sociétés, à leur évolution, à leurs structures humaines, à des groupes sociaux particuliers (la corporation, la commune, etc.), engagent nécessairement un certain sens philosophique des concepts de coopération et d'association. La signification du concept d'association chez Durkheim et Weber requiert alors de l'épistémologue un discernement entre les divers champs du savoir, les méthodes et les objets qui ont été mobilisés par l'auteur pour aboutir à telle ou telle conclusion.

\section{Organisme et association chez Durkheim}

\subsection{Préliminaires: Spencer et l'évolution des sociétés}

Parler de l'association chez Durkheim requiert un petit détour chez un écrivain qui, vers 1875 , élabore une approche pluridisciplinaire des sociétés, sans être véritablement sociologue : il s'agit d'Herbert Spencer. Comme d'autre auteurs du XIX ${ }^{\mathrm{e}}$ siècle, Spencer s'appuie sur les découvertes récentes des biologistes pour fonder sa propre thèse en forme d'organicisme social ${ }^{1}$. Il utilise le concept de division du travail pour faire le lien entre organisme et

1. Spencer, Herbert, Essais de morale, de science et d'esthétique, IIIè partie, Paris, Alcan, 1875. 
société, ce que d'autres ont tenté de faire également à la même époque. Le biologiste Milne-Edwards (1857) avait fait de la division du travail le moteur de la spécialisation des organes dans l'être vivant. Spencer utilise la même idée, mais en la replaçant dans une tendance évolutive des sociétés ${ }^{2}$. Ainsi, chez Spencer, le thème de la division du travail est subordonné à la tendance évolutive de type néodarwinien à l'organisation structurelle (ce qui n'était pas le cas chez Milne-Edwards). Chez Spencer, la société est un être vivant, et, comme pour l'être vivant, sa loi interne est de croître en organisation et en activité. Comment n'a-t-il pas vu les limites de son raisonnement : la société peut-elle être mise au même degré d'intégration et d'organisation que le corps de l'homme? La division du travail, point commun entre la société et le vivant, permet donc à Spencer de raisonner l'évolution sociale comme celle du vivant. Il replace la succession des formes des sociétés humaines dans une théorie d'évolution. Pour Herbert Spencer, la société la plus évoluée est celle où la coopération des individus sera la plus parfaite, la plus spontanée, réalisant le corps social sans avoir besoin d'un pouvoir contraignant et fort. En d'autres termes, la libre coopération des individus pour le développement social sera telle que le corps de société qui en sera le fruit s'harmonisera le mieux possible avec la vie individuelle à travers une très grande spécialisation des fonctions et une compétitivité accrue. Cela se comprend aisément : plus les fonctions sociales sont spécialisées, plus elles tendent à correspondre à tel ou tel individu, et l'individu tend à s'identifier à elles. Spencer écrit :

La forme de société vers laquelle nous progressons, je tiens que ç'en est une où le gouvernement sera réduit au minimum, et la liberté individuelle forte au plus haut degré ; la nature humaine aura été tellement modelée par l'accoutumance sociale, et rendue si propre à la vie en commun, qu'elle aura besoin de peu de force restreignante extérieure ; ce sera une société dans laquelle le citoyen ne tolérera aucune restriction de sa libre activité sauf ce qui est indispensable pour maintenir la liberté égale des autres, une société où la coopération spontanée qui a développé notre système industriel, et qui le développe à un rythme croissant, produira des organes pour l'accomplissement de toutes les fonctions sociales, et ne laissera à l'organe gouvernemental que la seule fonction de maintenir les conditions de l'acte libre, conditions qui rendent possible la coopération spontanée. Ce sera une société enfin où la vie individuelle sera poussée au plus haut degré compatible avec la vie en société, et où la vie sociale n'aura d'autre fin que de maintenir la sphère la plus complète de vie individuelle ${ }^{3}$.

Toutefois, l'optimisme de Spencer nous paraît quelque peu démesuré. S'il a justement prévu un développement des fonctions spécialisées dans la société et la réduction de l'appareil d'État dans le système libéral, on ne voit pas comment il tire de ce système une coopération spontanée, encore moins un

2. Milne-Edwards, Henri, Leçons sur la physiologie et l'anatomie comparée des hommes et des animaux, tome I, Paris, Masson, 1857.

3. Spencer, Herbert, Essais, IIIè partie, pp. 72-73. 


\section{$354 \cdot$ Philosophiques / Automne 2000}

corps de société en cohésion. Spencer développe une telle théorie, en partant de l'évolution parfois constatée, parfois supposée des sociétés, mais sans véritable analyse des faits sociaux (ce que fera Durkheim). Il manque donc à une telle approche un certain réalisme qui parte de l'expérience politique ou sociale, sans la projeter d'emblée dans une théorie d'origine biologique. Se situant dans la perspective du progrès des sociétés par l'organisation, Spencer ne discerne pas bien les conditions de la cohésion du corps social, parce que celles-ci dépassent justement cette notion d'organisation par une exigence éthique et politique. Ainsi, il ne traite pas réellement du concept d'association dans toute son extension. Cependant, les associations ne sont pas entièrement absentes de son oeuvre. Traitant de l'activité intérieure des sociétés, Spencer aura une vision très moderne des interrelations entre les divers organismes de la vie sociale.

Son activité intérieure, qui se développe au moyen de la diversification des fonctions, et d'une adaptation réciproque toujours plus efficace de parties toujours plus subdivisées et plus particularisées, ne réclame pas d'unique régulateur central, élabore au contraire en dehors de l'organe gouvernemental, des organes régulateurs distincts et nombreux comme les marchés de matière première ou de valeurs, les chambres de composition bancaire, les syndicats et associations diverses ${ }^{4}$.

\subsection{Quelle est la méthode sociologique chez Durkheim?}

Dans la même perspective, Durkheim se sert de la vision saint-simonienne sur les sociétés de type industriel : c'est le fait du progrès qui appelle des sociétés plus parfaites, parce que plus organisées ; l'organisation appelle la dépendance des parties entre elles, et à l'égard de l'ensemble (plus que l'exercice d'une autorité centrale). Spencer regardait la société en interaction avec les organes intermédiaires et les individus. La méthode sociologique que met en place Durkheim, nous introduit à un regard spécifique sur la société, comme entité nouvelle, existant par son être propre. Se plaçant au plan de l'observation des faits (et non pas au plan d'une éthique humaine), Durkheim observe que les individus recherchent des fins très diverses, malgré une nature commune : "Chaque individu a son histoire, quoique les bases de l'organisation physiques et morales, soient les mêmes chez tous ${ }^{5}$. Le finalisme, au sens de Durkheim, est donc connexe au règne de la contingence. De fait, Durkheim nous aide à considérer que même s'il existe une ou des finalités humaines morales universelles, elles ne sont que très rarement déterminantes si on se place au plan social. En effet, tous les individus ne recherchent pas de la même façon le même bien. Les faits éthiques individuels sont donc multiples : "que de voies différentes peuvent être, et sont effectivement

4. De Juvenel, Bertrand, Du pouvoir, Paris, Hachette, Pluriel, 1972, pp. 101-102.

5. Durkheim, Émile, 1895, Les règles de la méthode sociologique, Paris, Flammarion, Champs, 1988, p. 187. 
suivies ». Or, Durkheim note que cette individualité de la recherche des fins contraste avec l'uniformité des phénomènes sociaux. "Quand on est entré en contact quelque peu avec les phénomènes sociaux, on est surpris au contraire, de l'étonnante régularité avec laquelle ils se reproduisent, dans les mêmes circonstances. Même les pratiques les plus minutieuses, et en apparence, les plus puériles, se répètent avec la plus étonnante uniformité ${ }^{6}$. Durkheim va donc se détacher d'une éthique des fins personnelles, qu'il ne peut pas mesurer au plan des réalisations, tant elles diffèrent dans leur individualité, pour réintégrer la morale au plan des conditions d'une dynamique de vie sociale. «La morale, c'est le minimum indispensable, la structure nécessaire, le pain quotidien, sans lesquelles les sociétés ne peuvent pas vivre ${ }^{7}$. Selon Durkheim, la solidarité est l'exemple type du dépassement de la morale individuelle par le point de vue social. Mais, de quelle solidarité veut-il parler ? Il peut s'agir d'une forme culturelle et organisationnelle de solidarité dans une société donnée : selon que l'on appartient à tel groupe, que l'on travaille dans telle branche, on est structurellement solidaire des autres membres du groupe dans les relations socioprofessionnelles. Dans ce cas, cette forme de solidarité intégrerait au plus la déontologie : le médecin généraliste travaillera en liaison avec tel pharmacien ou tel médecin spécialiste vis à vis desquels il respectera certains codes. Ou encore, quelle que soit la qualité morale des relations dans une famille, elle forme une structure relativement solidaire.

Donc, en tant qu'elle produit des effets sociaux mesurables et reproductibles, elle est un «fait social ", elle "relève de la sociologie ${ }^{8}$. Durkheim écrit encore :

Si tant de moralistes et de psychologues ont pu traiter la question sans suivre cette méthode, c'est qu'ils ont tourné la différence. Ils ont éliminé du phénomène tout ce qu'il y a de spécifiquement social, pour n'en retenir que le germe psychologique dont il est le développement. Il est certain, en effet, que la solidarité, tout en étant un fait social au premier chef, dépend de notre organisme individuel... La solidarité, au plan psychologique, est une virtualité intangible, qui n'offre pas prise à l'observation. Pour qu'elle prenne une forme saisissable, il faut que quelques conséquences sociales la traduisent au dehors. ${ }^{9}$.

Cette observation n'est pas très juste parce que la solidarité au sens moral suppose la vertu et le développement de certaines dispositions psychologiques altruistes dont on peut juger, que l'on peut discuter. Quoi qu'il en soit, nous tenons donc à ce niveau tout ce qui fera l'originalité, à partir de Durkheim, de la méthode sociologique :

6. Durkheim, Émile, Ibid., p. 187.

7. Durkheim, Émile, 1893, La division du travail social, Paris, P.U.F, Quadrige, 1991, p. 14.

8. Durkheim, Émile, La division du travail social, p. 31.

9. Durkheim, Émile, Ibid., p. 32. 
- Au plan des réalisations tangibles, les fins éthiques personnelles ne sont pas objets de mesure même si elles influent heureusement dans l'ordre de la dynamique sociale.

- Il en est de même de tout l'ordre humain personnel et psychologique qui ne peut pas, à lui seul, résumer les caractères propres de l'ordre social. "Une explication psychologique des faits sociaux ne peut pas manquer de laisser échapper tout ce qu'ils ont de spécifique, c'est à dire de social $»^{10}$.

- Les faits sociaux ne sont pas non plus explicables dans l'ordre historique, par une tendance uniforme de développement (comme le voudrait Marx). En effet, "cette tendance, qui est censée être la cause de ce développement, n'est pas donnée ${ }^{11}$; toutefois, Durkheim réintégrera l'ordre historique (comme l'ordre moral), au niveau des conditions du dynamisme et de la cohésion sociale. Le « développement graduel » des sociétés, est en effet mesurable, par la « division du travail », constituant la « solidarité sociale. »

Nous commençons donc à entrevoir, que le principe de causalité, chez Durkheim, s'inscrit dans l'ordre du raisonnement expérimental. Il s'agit, en fait, de rechercher une relation causale, à partir des faits sociaux, relation qui "résulte de la nature des choses ${ }^{12}$. Ce déterminisme positif de Durkheim s'établit, en parallèle d'autres grands textes du XIX ${ }^{\mathrm{e}}$ siècle, comme ceux de Spencer et de Comte, ou encore "L'introduction à la médecine expérimentale ", de Claude Bernard, bien qu'il ne cite jamais ce dernier. Pour Durkheim, les faits sociaux permettent de découvrir une cause naturelle dans la réalité. Leur régularité, leur caractère positif plaident en faveur de la spécificité de la société, et de sa réalité propre. Durkheim croit découvrir que "le social est une réalité sui generis, qu'il est doté d'une vie propre ${ }^{13}$. N'est-ce pas une reprise des idées de Saint-Simon dans la « Physiologie sociale » ? Berthelot écrit encore : "Le naturalisme [de Durkheim], ancre du côté de l'être, et non plus seulement de celui du connaître, l'idée d'une réalité propre de la société. Celle-ci n'est ni un développement ou une expression de l'humain (Spencer, Comte), ni une création résultant d'un contrat social (Hobbes, Rousseau) ${ }^{14}$.

Au plan épistémologique, le reproche que l'on peut faire à Durkheim porte sur la possibilité de passer des faits sociaux à un milieu social vu comme individualité. L'application de la méthode expérimentale aux données d'ordre social, découvre au delà des faits, des relations constantes et significatives,

10. Durkheim, Émile, Les règles de la méthode sociologique, p. 199.

11. Durkheim, Émile, Ibid., p. 210.

12. Durkheim, Émile, Ibid., p. 219.

13. Berthelot, Jean-Michel, Les règles de la méthode sociologique ou l'instauration du raisonnement expérimental en sociologie, in "Les règles de la méthode sociologique ", É. Durkheim, 7-67, Champs, Flammarion, Paris, 1988, p. 40.

14. Berthelot, Jean-Michel, Ibid., p. 41. 
saisies comme nécessaires. Ce moment, analogue à l'induction des lois en sciences expérimentales, appelle chez Durkheim, la conviction suivante : la constance et la régularité des phénomènes sociaux, référés à des causes, apportent la preuve de l'unité de la vie sociale, de cet organisme individuel qu'est la société. Ce passage est intellectuellement contestable, parce que Durkheim cherche à raisonner au niveau d'un grand vivant hypothétique, la société, à partir de la saisie de relations interindividuelles. L'originalité du social, comme expression vitale, est découvert dans toute sa force ; mais, rejetant l'idée de l'expression d'une dimension particulière de l'humain, Durkheim veut induire à partir de la multiplicité des relations entre les individus - relations qui sont aussi des actes vitaux - l'unité du vivant sociétal. Même si ces multiples relations donnent lieu à des phénomènes constant et réguliers, on ne voit pas comment on peut découvrir au terme la société comme une totalité existentielle. Certains formes de coopération, ou au contraire certaines formes de lutte entre les hommes peuvent donner lieu aux mêmes effets sans que, pour autant, la société soit une réalité une, un être spécifique. Ce type de raisonnement ne semble pas validable. Ne doit-on pas aussi, en toute rigueur, distinguer l'harmonie des phénomènes sociaux et l'association des consciences individuelles ? Et que dire du lien entre les découvertes de la sociologie d'une part, l'anthropologie et la philosophie politique d'autre part ? Ce lien, que Durkheim rejette, n'est-il pas d'actualité dans les mutations considérables que connaissent nos sociétés ? En effet, le problème de la prise en compte des besoins et des projets des personnes fait repenser l'autonomie du social. Par ailleurs, l'éclatement des sociétés des pays occidentaux est tel que, s'il fallait individualiser aujourd'hui comme un organisme vivant, un groupe humain, ce serait l'organisation (association, entreprise ou collectivité locale), qui serait probablement l'unité de vie et de durée.

\subsection{Au fondement de l'association, l'analogie entre la société et le vivant.}

C'est parce que Durkheim rejette cette subordination du social à l'humain, et qu'il veut découvrir la cause de la cohérence globale des relations vitales et sociales découvertes, qu'il veut induire, à partir de l'association des individus en société15, l'existence d'une individualité vivante nouvelle, et qu'il fait reposer cette induction sur l'analogie biologique. En effet, le caractère naturel et vivant de la dimension sociale, l'idée durkheimienne d'une unique causalité des faits sociaux, appellent d'elles-mêmes le recours à cette analogie, dont il faut interroger la validité.

Cette analogie s'applique éminemment aux questions de la division du travail, et de la " solidarité organique ». Dans les sociétés où règne une solidarité organique, et dans lesquelles le principe de division du travail a atteint son évolution optimale, chaque organe social accomplit une fonction spécia- 


\section{$358 \cdot$ Philosophiques / Automne 2000}

lisée et déterminée, dans l'interdépendance et la complémentarité. Durkheim écrit, dans La division du travail :

Les éléments sociaux ne sont ni juxtaposés linéairement comme les anneaux d'un annelé, ni emboîtés les uns dans les autres, mais coordonnés et subordonnés les uns aux autres autour d'un même organe central qui exerce sur le reste de l'organisme une action modératrice. Cet organe lui-même n'a plus le même caractère que dans le cas précédent (la solidarité mécanique), car, si les autres dépendent de lui, il en dépend à son tour. Sans doute, il a bien encore une situation particulière et, si l'on veut, privilégiée ; mais elle est due à la nature du rôle qu'il remplit, et non à quelque cause étrangère à ses fonctions, à quelque force qui lui est communiquée du dehors.

Pour Durkheim, les évolutions sociales montrent que toute société est appelée à passer d'une organisation politico-sociale segmentaire ${ }^{16}$, à une organisation beaucoup plus "évoluée ", basée sur le milieu professionnel, impliquant la spécialisation et la division du travail, dans la coopération ${ }^{17}$. Mais, cette évolution sociale implique une tendance à l'organisation dont on ne découvre pas en réalité la cause efficiente. Cette dernière reste très idéale, sinon idéologique. En effet, de l'analyse des faits sociaux, on retomberait (comme chez SaintSimon, Comte ou Spencer) sur la tendance (lamarckienne) de la vie à la complexification. Or, dans le domaine socio-politique, peut-on envisager une telle tendance à l'ordre et à la cohésion sociale, tendance qui semble spontanée ? Ici, le sociologue semble donc oublier les rôles de l'autorité et de la liberté dans le progrès des sociétés. Cet oubli du rôle de l'autorité, en matière d'évolution et de progrès, était aussi l'objet d'un reproche de Huxley à Spencer. On pense encore à une remarque de Charles Maurras, dans l'enquête sur la monarchie: "C'est une des plus fortes maximes de M. de la Tour du Pin, que l'ordre ne naît pas spontanément dans la société. Une autorité le précède ; et elle l'engendre. Sans cette autorité génératrice et directrice, les meilleures dispositions peuvent se corrompre ou se retourner contre le salut public ${ }^{18}$.

Une autre analyse intéressante et contemporaine à Durkheim est celle de Duthoit. Pour Eugène Duthoit, comme pour Durkheim, la division du travail social, au sens de spécialisation est connexe à un accroissement de solidarité :

Tandis que le travail se divise et se morcelle, par l'effet d'une spécialisation qui étend chaque jour ses conquêtes, les travailleurs voués aux tâches les moins semblables ont conscience d'être unis par les liens d'une étroite solidarité. Les hommes tendent de plus en plus à se différencier les uns des autres ; ils ont des aptitudes particulières qui les poussent à se spécialiser, mais aussi des besoins différents qui les incitent à se rapprocher, et à pratiquer un échange mutuel de

16. Durkheim, Émile, La division du travail social, p. 161.

17. Durkheim, Émile, Ibid., pp. 93-94.

18. Maurras, Charles, 1909, L'enquête sur la Monarchie, Paris, Nouvelle librairie nationale, 1916, p. 226. 
services. Plus les aptitudes individuelles sont accusées, plus les échanges sont fréquents et la solidarité grandit dans la même proportion que la division du travail social ${ }^{19}$.

Chez le chrétien Duthoit, comme chez l'agnostique Durkheim, cette situation crée des conditions favorables à l'établissement de la corporation. Mais, là, leurs chemins se séparent, la corporation au sens de Durkheim étant beaucoup plus liée à l'État...

À partir du moment où coopérer, c'est se partager une tâche commune, la tendance observée dans le sens de la spécialisation permet, selon Durkheim, de prévoir « qu'un jour viendra où toute notre organisation sociale et politique aura une base exclusivement, ou presque, professionnelle ${ }^{20}$. Cette base professionnelle de l'organisation socio-politique, correspond à ce que Saint-Simon imagine comme étant l'association fraternelle des producteurs ${ }^{21}$. Durkheim maintient dans la société le primat des forces morales, auxquelles sont subordonnées les actions économiques. Pour Durkheim, si Saint-Simon cherche à équilibrer les dangers de domination des nouveaux pouvoirs sociaux et économiques par une morale de l'amour au sens de philanthropie, voire par une sorte de nouvelle religion universelle, ce ne sont pas des moyens de réalisation de l'ordre social, mais des thèmes annexes qui n'ajoutent rien. C'est que, au coeur même de l'organisme social et de la division du travail, la morale reste, "le minimum indispensable » à pratiquer par l'individu ${ }^{22}$. Elle se situe surtout au niveau des groupes, et est donc corrélative à la division du travail : c'est ce que Saint-Simon n'a pas vu. Durkheim écrit, à ce propos, dans Leçons de Sociologie :

Une morale est toujours l'oeuvre d'un groupe et ne peut fonctionner que si ce groupe la protège de son autorité. Elle est faite de règles qui commande aux individus, qui les obligent à agir de telle ou telle manière, qui imposent des bornes à leurs penchants et leur défendent d'aller plus loin. Or, il n'y a qu'une puissance morale, et par conséquent commune, qui soit supérieure à l'individu, et qui puisse légitimement lui faire la loi, c'est la puissance collective. Dans la mesure où l'individu est abandonné à lui-même, dans la mesure où il est affranchi de toute contrainte sociale, il est affranchi aussi de toute contrainte morale. La morale professionnelle ne saurait se soustraire à cette condition de toute morale. Puisque donc la société dans son ensemble s'en désintéresse, il faut donc qu'il y ait dans la société des groupes spéciaux au sein desquels elle s'élabore, et qui veillent à la faire respecter. Ces groupes, c'est et ce ne peut être que les groupes formés par la réunion des mêmes individus de la même profession, ou groupes professionnels ${ }^{23}$.

19. Duthoit, Eugène, Vers l'organisation professionnelle, Reims, Action populaire, 1910 , p. 311.

20. Durkheim, Émile, La division du travail social, p. 167.

21. Cf. Ansart, P., Marx et l'anarchisme, Première partie, "Saint-Simon », Paris, P.U.F, 1969.

22. Durkheim, Émile, La division du travail social, p. 14.

23. Durkheim, Émile, Leçons de sociologie, 1950, Paris, P.U.F, Quadrige, 1990, p. 46. 
On retrouve dans un tel texte une acception propre à Durkheim du concept de morale, ainsi que nous l'avions déjà signalé. La morale personnelle, relative au bonheur, à l'amitié, à la vérité, etc., est absente. Seul subsiste le minimum de dispositions morales, voire déontologiques pour permettre à la société de fonctionner. Généralement, ces dispositions peuvent se traduire par un code de négations. C'est le cas aujourd'hui dans les codes de déontologie des entreprises, par exemple, où bien souvent l'éthique prônée est inframorale (en ce qu'elle ne mobilise pas les plus hautes qualités morales de l'homme). Ainsi, il semblerait que la morale professionnelle aille plus loin que la coopération des hommes producteurs, dans l'activité commune de la société saint-simonienne. Pour Durkheim en effet, l'activité de coopération la plus élevée dans les groupes sociaux, c'est l'élaboration de la morale professionnelle, qui va régir ensuite les autres activités de coopération.

La tendance à l'accroissement de la solidarité organique, et donc, à la professionnalisation, découverte à partir des faits de société, semble vue par ailleurs au sens restrictif d'accomplissement des tâches utiles, immédiatement mesurables. C'est ce qui fait la limite de son raisonnement : les fonctions sociales d'ordre éducatif, mais aussi culturel, artistique, spirituel... peuvent-elle être prises en compte dans un tel schème de société? Dans d'autres oeuvres, Durkheim insiste beaucoup sur l'éducation, mais son caractère particulier, non immédiatement productif, est-il compatible avec la vision de la société que nous venons d'évoquer? L'organicisme social semble, en apparence, réduire les fonctions et les fins sociales au développement de la vie socioprofessionnelle, et à la prospérité économique. Est-il réaliste? Estil réellement analogique au niveau de la vie? Pour répondre à ces questions, il est nécessaire d'examiner l'autre terme de l'analogie : l'organisme biologique. "La même loi ", sous-entendue d'organisation et de division du travail, écrit Durkheim «préside au développement biologique ${ }^{24}$. À ce niveau, c'est essentiellement l'oeuvre d'Edmond Perrier, et une certaine inspiration saint-simonienne, qui intéressent le sociologue. Mais, comme Perrier, Durkheim s'inspire aussi du regard de Milne-Edwards, sur la division physiologique du travail dans l'organisme ${ }^{25}$.

La première voie de formation d'une individualité nouvelle établie par Perrier, est la colonie des invertébrés. Commentant la définition de Perrier dans «Le transformisme » : « Toute colonie dont les membres sont en continuité de tissu, est en réalité un individu ", Durkheim écrit que "ce qui caractérise l'individualité d'un agrégat quelconque, c'est l'existence d'opérations effectuées en commun par toutes les parties. Or, entre les membres de la colonie, il y a mise en commun des matériaux nutritifs, et impossibilité de se mouvoir autrement que par des mouvements ensemble, tant que la colonie n'est pas dissoute; il y a plus ; l'oeuf issu de l'un des segments associés, reproduit, non

24. Durkheim, Émile, La division du travail social, p. 167.

25. Cf. aussi à ce sujet, Séris, Jean-Pierre, La division du travail, Paris, Vrin, Pré-textes, 1994. 
ce segment, mais la colonie entière dont il faisait partie ${ }^{26}$. Citant « Les colonie animales », Émile Durkheim affirme l'existence, dans le monde animal, d'une individualité qui se produit en dehors de toute combinaison d'organes ${ }^{27}$. C'est l'individualité de la colonie qui constituait à l'époque le concept central de l'oeuvre d'Edmond Perrier. Selon ce zoologiste, les individus les plus simples tendaient à s'agréger en colonies selon une loi naturelle de complexification et de fusion ; au terme de l'évolution, seuls les être supérieurs avaient acquis une individualité biologique, au delà de la colonie. Selon le sociologue, cette individualité correspond au type segmentaire des sociétés dans lesquelles la division du travail reste primaire. Dans ces sociétés segmentaires, l'association des individualités élémentaires est de l'ordre de la juxtaposition anatomique, comme chez les organismes inférieurs. Durkheim écrit, dans les « Règles » de la méthode sociologique, au sujet de la « horde » :

c'est un agrégat social, qui ne comprend et n'a jamais compris, en son sein, aucun autre agrégat élémentaire, mais qui se résout immédiatement en individus. Ceux-ci ne forment pas, à l'intérieur du groupe total, des groupes spéciaux et différents du précédent; ils sont juxtaposés anatomiquement. On conçoit qu'il ne puisse pas y avoir de société plus simple ; c'est le protoplasme $\mathrm{du}$ règne social, et par conséquent, la base naturelle de toute classification.

Le problème posé par cette analogie est qu'elle ne s'appuie immédiatement que sur "l'économie » de la vie biologique. Le point commun par excellence devient ce qui touche le "budget de l'organisme » ${ }^{28}$. Mais, une telle analogie vise, en fait, la constitution de l'unité vivante comme telle. En effet,

de même que le type segmentaire s'efface à mesure qu'on avance dans l'évolution sociale, le type colonial disparaît à mesure qu'on s'élève dans l'échelle des organismes. Déjà entamé chez les annelés, quoique encore très apparent, il devient presque imperceptible chez les mollusques... Nous n'avons pas à montrer les analogies qu'il y a entre le type qui remplace le précédent et celui des sociétés organiques. Dans un cas comme dans l'autre, la structure dérive de la division du travail ainsi que de la solidarité29.

L'idée de l'effacement progressif de l'organisation segmentaire est repris du double point de vue sociologique et biologique :

Tant que chaque segment a sa vie qui lui est particulière, il forme une petite société dans la grande et a, par conséquent, en propre ses organes régulateurs, tout comme celle-ci. Mais, leur vitalité est nécessairement proportionnelle à l'intensité de cette vie locale; ils ne peuvent donc pas manquer de s'affaiblir

26. Durkheim, Émile, La division du travail social, p. 167.

27. Perrier, Edmond, 1881, Les colonies animales et la formation des organismes, 2 è édition, Paris, Masson, 1887, p. 778.

28. Durkheim, Émile, Les règles de la méthode sociologique, p. 190.

29. Durkheim, Émile, La division du travail social, p. 169. 
quand elle s'affaiblit elle-même. Or, nous savons que cet affaiblissement se produit avec l'effacement progressif de la structure segmentaire ${ }^{30}$.

A ce niveau, nous sentons comme il est contestable de transposer la centralisation du vivant de vie biologique à la société : Qu'en est-il de la vitalité des corps intermédiaires, comme contribuant à la vie commune de l'ensemble? Durkheim poursuit :

Il peut se produire entre eux (les organes autonomes) une coalescence du même genre, mais non entre eux et lui ; ou du moins, s'ils sont soumis à l'action des centres supérieurs, ils en restent distincts. Chez les vertébrés, le système cérébro-spinal est très développé, il a une influence sur le grand sympathique, mais il laisse à ce dernier une large autonomie ${ }^{31}$.

L'autonomie des corps intermédiaires est donc préservée, dans une certaine mesure. Notons ici que :

Durkheim introduit en sociologie, la célèbre loi de coalescence de Dugès $(1831)^{32}$.

Il maintient l'autonomie de fonctions régulatrices particulières (l'économie), à l'égard de l'État, intégrant ainsi son argumentation dans un point de vue biologique.

Il semble néanmoins, réduire l'autonomie des corps intermédiaires les uns vis à vis des autres. Au plan biologique, le phénomène de symbiose s'établit sur une interrelation particulière, irréductible, qui produit l'autonomie. Au plan sociologique aujourd'hui, le phénomène associatif semblerait prendre de l'importance au plan de l'initiative et de l'autonomie, l'État jouant un rôle de régulateur général, et non plus un rôle analogue au " cerveau ». Les rôles d'initiatives des organismes intermédiaires et le renouvellement de la notion d'État remettent-ils en cause une telle application de l'analogie biologique à l'ensemble de la société ?

\subsection{L'association chez Durkheim entre groupe secondaire et État organisateur.}

Dans l'analyse que nous faisons de l'oeuvre de Durkheim, il ne faudrait cependant pas sous-estimer le rôle des groupes intermédiaires. Il existe, chez Durkheim, un équilibre entre individus, corps intermédiaires et État, qui est subtil, et qu'il importe de ne pas occulter. En effet :

Au sujet de l'individu, sa perfection morale est fondamentale dans la sociologie durkheimienne ; même si son autonomie est relative, il n'est pas pour autant absorbé dans l'organisme social. J.-C. Filloux écrit :

30. Durkheim, Émile, Ibid., p. 201.

31. Durkheim, Émile, Ibid., p. 202.

32. Dugès, Antoine, Mémoire sur la conformité organique, Montpellier, Ricard, 1831. Voir aussi Durkheim, Émile, Les règles de la méthode sociologique, p. 179. 
Pour Tocqueville, la perfection de l'individu est la fin de toute association politique, de telle sorte que sa théorie de l'individualisme est d'abord interprétation critique et dépassement de l'individualisme libéral, dans la perspective d'une révélation des valeurs de la personne, c'est à dire d'authenticité, aussi bien de l'homme que de la vie sociale. Durkheim continue cet effort tocquevillien pour définir le type d'individualisme qui dépasse ses déviations asociales, qui prennent au moment où Durkheim écrit, le nom d'économisme, d'utilitarisme, d'anarchisme ${ }^{33}$.

Au plan des corps intermédiaires, on trouve, comme nous l'avons dit, une autonomie relative conditionnant (paradoxalement) l'unification progressive du tout. J.-C. Filloux écrit :

D'une façon générale, le modèle durkheimien s'inscrit dans le mouvement qui pose, d'une manière ou d'une autre, la thèse du pluralisme de l'autorité ou du pouvoir, où l'on trouve Tocqueville, Proudhon, et bien entendu Renouvier. Pour faire sortir la liberté au sein même d'une société démocratique qui contient en germe l'anarchie et la servitude, Tocqueville conférait - comme Montesquieu - une partie des attributions du pouvoir à des corps secondaires... Proudhon cherchait à provoquer une limitation de l'État par les groupes, pour faire de l'autorité de l'État fédéral, non un pouvoir politique, mais un lieu de rencontre des intérêts subalternes à la confédération. Renouvier de son côté, proposait au moment où Durkheim élaborait sa pensée, de développer une décentralisation du pouvoir par l'autonomie de groupes locaux, tels que les communes et la formation de libres associations entre producteurs et consommateurs, de coopératives, de groupements de travailleurs ${ }^{34}$.

Et l'auteur ajoute que l'on retrouve les groupes secondaires et les associations chez Durkheim, mais que « le principe de pouvoir pluraliste chez Durkheim s'appuie sur l'analyse psychologique et sociologique du pouvoir social, tel qu'il sourd de la vie de groupe. "En effet, à partir de ces groupes, se constitue une force morale commune, mais aussi le risque d'une trop grande autonomie. Il faut donc concilier l'existence de l'État, représentant et organisant (au sens fort) la société totale d'une part, et celle des groupes secondaires qui permettent l'expression et la libération de l'individu.

Dans les Leçons de sociologie, Durkheim raisonne en termes d'équilibre : si l'autonomie relative des groupes locaux est indispensable pour laisser du champ au développement des individus, le rôle de l'État s'étend à toutes les fonctions de la vie sociale pour garantir et permettre la vie individuelle contre toute tyrannie arbitraire. Durkheim écrit :

On peut dire que c'est l'État qui a soustrait l'enfant à la dépendance patriarcale, à la tyrannie domestique, c'est lui qui a affranchi le citoyen des groupes féodaux, plus tard communaux, c'est lui qui a affranchi l'ouvrier et le patron de la tyrannie corporative... La fin que cette conception assigne ainsi à

33. Filloux, Jean-Claude, Durkheim et le Socialisme, Genève, Droz, 1976, p. 146. 34. Filloux, Jean-Claude, Ibid., pp. 239-240. 
l'État, les individus peuvent la comprendre ainsi que les rapports qu'elle soutient avec eux. Ils peuvent y collaborer en se rendant compte de ce qu'ils font, du lieu où va leur action, parce que c'est d'eux-mêmes qu'il s'agit. Ils peuvent même le contredire, et même par là se faire les instruments de l'État, puisque c'est à les réaliser que tend l'action de l'État. Et pourtant, ils ne sont pas comme le veut l'école individualiste utilitaire, ou l'école kantienne, des touts qui se suffisent à eux-mêmes, et que l'État doit se borner à respecter, puisque c'est par l'État et par lui seul qu'ils existent moralement ${ }^{35}$.

Cette dernière phrase est assez terrible. Finalement, à travers une dialectique subtile, on est ramené du rôle dynamique des associations au rôle premier de l'État. Il est centre de pensée et centre de vie, centre d'organisation. La vie morale elle-même est reçue de l'État, ce qui parait très ambigu et assez terrifiant ! L'État durkheimien est bien le « cerveau », le « foyer » de pensée sociale et de "vie mentale » qui renouvelle la société (tout en laissant jouer, dans une certaine mesure, le principe de subsidiarité). Il n'est pas, en réponse à la question posée ci-dessus, le garant général des équilibres libéraux. Toute l'oeuvre de Durkheim tendant à faire d'un groupe social, une réalité et un organisme spécifique, il est logique que la " personnalité » propre de l'État l'emporte sur les autres, d'où la difficulté de bien définir les équilibres, y compris au niveau moral.

\subsection{Vers une synthèse corporative.}

C'est dans l'ordre corporatif que va éclater la nouvelle synthèse durkheimienne. Puisque les groupes secondaires sont indispensables à l'expression et à la communion des individus, et que l'État reste le centre de l'organisation du tout social, la synthèse dialectique et logique aboutit à l'organisation corporative. Pour Durkheim, dans La division du travail, ou dans les leçons de sociologie, il s'agit d'aller au delà de l'ancienne corporation, qu'on ne doit même pas songer à transformer. La décentralisation sociale ne peut être pour Durkheim que professionnelle (ce qui rejoint les thèmes principaux de La division du travail, mais qui est en soi contestable). L'organisation de la corporation implique, comme la société dans son ensemble, un organe central (conseil d'administration) et des organes secondaires. Mais, la corporation étant elle-même intégrée dans l'État, les conseils corporatifs envoient des délégués à une assemblée politique qui fait partie de l'État. Un tel système fait un peu penser aux corps intermédiaires de Montesquieu ou aux assemblées de Turgot. Tout le problème est de savoir jusqu'où peut aller l'interrelation organique du pouvoir de l'État et du pouvoir de la corporation. Filloux écrit à propos de cette organisation prônée par Durkheim :

Les problèmes concernant la vie professionnelle, notamment dans la sphère économique, passent aisément dans la sphère des décisions gouvernementales :

35. Durkheim, Émile, Leçons de sociologie, p. 99. 
le pouvoir des groupes professionnels trouve un moyen pour s'exercer et contrebalancer le pouvoir du groupe Étatique. Inversement, l'État peut imposer par l'intermédiaire des conseils, qui ainsi participent de l'action de l'État, des règles générales précisant la manière dont sont susceptibles de s'articuler les diverses corporations ${ }^{36}$.

On a bien, au niveau des corporations, la synthèse dialectique que nous évoquions ci-dessous, dans le cadre épistémologique de "l'organicisme », et on n'évite pas l'ambivalence dans l'ordre des enjeux de pouvoir.

Revenons à l'analogie biologique : dans La division du travail, c'est plutôt sur l'unification progressive du vivant à travers l'évolution, que porterait véritablement l'analogie au sens de Durkheim. Il semble toutefois légitime de remettre en cause cette analogie, dans la mesure où le vivant social n'est jamais une réalité totalement organique ; qu'il n'existe pas en vue de sa propre conservation, mais de la conservation des individus et des organismes intermédiaires qui le constituent ; que le naturalisme et le vitalisme qui justifient son existence même font partie du climat épistémologique de la fin du $\mathrm{XIX}^{\mathrm{e}}$ siècle. D'ailleurs, cette analogie, même si elle dégage un terme commun, respecte-t-elle la diversité des domaines? Elle semble parfois transposer formellement les observations et les modèles biologiques en sociologie. La multiplicité des approches de l'être vivant, au $\mathrm{XX}^{\mathrm{e}}$ siècle, souligne le comportement tout autre de l'organisme sociétal . L'approche évolutive proposée par Edmond Perrier supposait l'association d'individualités élémentaires ; l'approche du Néodarwinisme, mettant en valeur l'équilibre "mutationsélection », pour un stock chromosomique, ne permet plus de garder les mêmes parallèles. Tributaire du contexte épistémologique de son temps et d'un certain regard (celui de la zoologie des invertébrés, et de l'anatomie), l'analogie de Durkheim ne tient pas face à la génétique moléculaire, qui mettrait en valeur dans le vivant tout le point de vue de l'individualité, et de l'autonomie. Par ailleurs, le but de Durkheim n'est-il pas plutôt à situer au niveau de l'applicabilité de la méthode expérimentale en sociologie?

Enfin, des textes de Durkheim montrent que c'est bien sur les concepts d'unification, d'association et de coopération que porte l'essentiel de la réflexion, et que ces concepts sont ouverts, chez Durkheim, à toute la plasticité et l'humanisation des faits sociaux. Dans La division du travail, Durkheim considère que la coopération entre les hommes, va bien au delà d'un équilibre contractuel d'échanges, exprimant une interdépendance économique. Il écrit :

Sans doute, quand les hommes s'unissent par le contrat, c'est que, par suite de la division du travail, ils ont besoin les uns des autres. Mais, pour qu'ils coopèrent harmoniquement, il ne suffit pas qu'ils entrent en rapport, ni même qu'ils sentent l'état de mutuelle dépendance où ils se trouvent. Il faut encore

36. Filloux, Jean-Claude, Durkheim et le Socialisme, p. 344. 
que les conditions de cette coopération soient fixées, pour toute la durée de leurs relations ${ }^{37}$.

Par ailleurs, la division du travail social se distingue de la division physiologique du travail " par un caractère essentiel. Dans l'organisme, chaque cellule a son rôle défini, et ne peut en changer. Dans la société, les tâches n'ont jamais été réparties d'une manière aussi immuable... » En réalité, Durkheim se sert du modèle et de la méthode découverts en sciences expérimentales, pour mettre en valeur la coopération et l'association, comme réalités que l'on peut établir scientifiquement, au plan humain. Pour Durkheim, il est clair que la mutuelle dépendance conditionne la coopération ; mais, cette dernière va plus loin au plan de l'engagement volontaire de l'homme : "Elle nous astreint à des obligations que nous n'avons pas contractées, au sens exact du mot, puisque nous ne les avons pas délibérées, ni même parfois connues par avance... Nous coopérons parce que nous l'avons voulu, mais notre coopération volontaire nous crée des devoirs que nous n'avons pas voulu ${ }^{38}$.

De plus, l'altruisme intervient fortement au niveau de la coopération. L'état de dépendance de l'individu à l'égard de la société, de la partie à l'égard du tout appelle la coopération, non plus entre individus seulement, mais entre les individus et la société.

La société apprend à regarder les membres qui la composent, non plus comme des choses sur lesquelles elle a des droits, mais comme des coopérateurs dont elle ne peut se passer, et vis à vis desquels elle a des devoirs. C'est donc à tort qu'on oppose la société qui dérive de la communauté des croyances à celle qui a pour base la coopération, en n'accordant qu'à la première un caractère moral, et en ne voyant dans la seconde qu'un groupement économique. En réalité, la coopération a, elle aussi, sa moralité intrinsèque. Il y a seulement lieu de croire, comme nous le verrons mieux dans la suite, que dans nos sociétés actuelles, cette moralité n'a pas encore tout le développement qui leur serait dès maintenant nécessaire ${ }^{39}$.

À ce sujet, G. de Terssac écrit :

En même temps qu'elle différencie les postes de travail, la division du travail amène la nécessité de les coordonner et, du même coup, oblige les individus à coopérer. La division du travail rend les individus qui réalisent les tâches dépendants les uns des autres : sont-ils pour autant solidaires? C'est cette question originelle que traite Durkheim (1893) pour qui la division du travail n'a pas seulement un rôle économique, mais aussi un rôle social, au sens où elle constitue un puissant facteur d'intégration sociale ${ }^{40}$.

37. Durkheim, Émile, La division du travail social, p 190.

38. Durkheim, Émile, Ibid., p. 192.

39. Durkheim, Émile, La division du travail social, p. 208.

40. Terssac, G. de, Organisation du travail et sociologie, Revue française de gestion, 1993, 90-100. 
Les déclarations d'Émile Durkheim sont une reprise de la sociologie de Spencer, affirmant qu'une société n'existe que par la coopération " consciemment instituée, qui suppose des fins d'intérêt public nettement reconnues ${ }^{41}$.

Dans l'État de solidarité organique, définissant l'exigence de coopération comme un "plus » dans l'ordre de l'efficience, et comme " réglée » par une morale, l'auteur s'interroge sur l'association des individus qui coopèrent. La division du travail semble, pour lui, induire des liens entre les membres, « liens qui s'entendent bien au delà des moments si courts où l'échange s'accomplit », et qui créent un "réseau d'obligations ${ }^{42}$. Dans les règles, Durkheim affirme que « le fait de l'association est le plus obligatoire de tous ». Même si «j'acquiesce », il demeure un caractère impératif et déterminé. Cependant, l'association, vue dans un certain regard psychologique, constitue bien "une réalité spécifique qui a ses caractères propres "43, une " combinaison des consciences ", une "individualité psychologique d'un genre nouveau ». L'idée de la nouvelle individualité n'est plus ici exploitée selon le parallélisme biologique, mais en fonction d'une continuité de phénomènes psychiques, d'ordre spécifiquement humain. On peut s'interroger sur l'affirmation "d'une agrégation des âmes individuelles ", agrégation qui donne naissance à un nouvel être psychique. C'est, d'après les tendances collectives, les "produits psychiques » de la vie sociale, que Durkheim croit pouvoir affirmer cette existence nouvelle. Il faudra tout l'effort de la psychosociologie et de la psychanalyse, pour discerner ce qui est d'ordre individuel, et ce qui est dû à des habitudes, ou à des pressions «de groupe ", dans les actes et les processus sociaux.

\section{Individu et association chez Max Weber.}

\subsection{Quelle méthode pour la « sociologie compréhensive »?}

Évoquer le concept d'association chez Max Weber n'est pas à première vue chose facile quand on sait que celui-ci écrivait en 1920, dans une lettre à R. Liefmann :

Si je suis finalement devenu sociologue, c'est essentiellement afin de mettre un point final à ces exercices à base de concepts collectifs dont le spectre rode toujours. En d'autres termes, la sociologie, elle aussi ne peut procéder que d'un, de quelques ou de nombreux individus séparés. C'est pourquoi elle se doit d'adopter des méthodes strictement individualistes ${ }^{44}$.

41. Durkheim, Émile, Les règles de la méthode sociologique, p. 114.

42. Durkheim, Émile, La division du travail social, p. 207.

43. Durkheim, Émile, Ibid., p. 196.

44. Weber, Max, 1920, in Raynaud Philippe, Max Weber et les dilemmes de la raison moderne, Paris, P.U.F, 1987, p. 93. 
Cependant le fait de prendre l'individu comme point de départ de la méthode sociologique n'exclut pas sa mise en association. Cela n'implique simplement que le renoncement à poser a priori l'organisme sociétal comme individualité supérieure et vivante. Par ailleurs, le regard sur le vivant est très présent chez Max Weber : l'individu n'est pas une entité abstraite, mais le sujet d'opérations vitales, qui va coopérer et interagir avec d'autres individus. L'oeuvre de Max Weber s'intègre donc tout à fait dans notre sujet, en tant que nous nous efforçons de comprendre les concepts d'individualité et d'association dans le prolongement d'activités vitales et de recherches de finalités propres à l'homme. Le problème consistera alors à bien cerner le point d'arrivée de la sociologie weberienne. À ce niveau, Weber évoque l'activité rationnelle par finalité ${ }^{45}$. Mais il n'est pas évident de comprendre, chez Max Weber, de quelle finalité il s'agit.

Dans " Essais sur quelques catégories de la sociologie compréhensive ", il est question, d'une part, d'activité « orientée subjectivement par finalité »et, d'autre part, d'activité « orientée judicieusement d'après ce qui est objectivement valable ${ }^{46}$. Ainsi, la sociologie compréhensive se base sur le comportement significatif de l'individu, comportement impliquant les deux types d'activité susnommées, qui ne sont pas incompatibles. La structure de la vie morale et psychologique serait donc un noyau donnant aux faits sociaux leur intelligibilité. En particulier, la « justesse » des fins et des moyens choisis par les individus permettent de comprendre un enchaînement causal donnant naissance à des structures sociales. Max Weber écrit encore dans « Essai sur quelques catégories de la sociologie compréhensive » :

Il y a lieu de parler de "causalité significativement adéquate", dans l'histoire de la logique, quand, à propos d'un ensemble subjectivement significatif de discussions sur des problèmes logiques, il «vient à l'esprit » du penseur une idée qui se rapproche du type de justesse de la " solution ». Il en est de même, en principe, d'une activité qui nous semble avoir spécifiquement une "cause significativement adéquate " du fait qu'elle s'oriente d'après la réalité "conforme à l'expérience ». Il s'en faut de beaucoup que, si le déroulement réel d'une activité se rapproche en fait très considérablement du type de justesse (un cas de rationalité par justesse réellement objectif), on doive nécessairement parler d'une concordance avec l'activité subjective rationnelle par finalité, c'est-à-dire celle qui s'oriente d'après des fins conçues de façon consciemment univoques et des moyens choisis comme consciemment adéquats ${ }^{47}$.

Une telle approche de la cohérence (intention-moyens-fins) dans le comportement moral permet de saisir l'approche weberienne en matière de communauté et d'association. On retrouvera dans l'association, d'une part,

45. Weber, Max, 1913, "Essai sur quelques catégories de la sociologie compréhensive ", in Essais sur la théorie de la science, Paris, Plon, 1992, pp. 303-364,

46. Weber, Max, Ibid., p.310.

47. Weber, Max, Ibid., pp. 311-312. 
une activité "subjectivement significative », et d'autre part, l'existence de buts objectifs impliquant un sens et des moyens à mettre en oeuvre.

Parvenus à ce point, observons que le manque de précision dans la réalité des fins poursuivies par les associations ne permet pas de donner une éthique sociale. Max Weber se situe au niveau d'une critique qui porte sur les rapports entre l'activité subjective et l'objectivement valable, la réalité saisie dans l'expérience. De ces rapports éthiques réalisés par l'individus naissent les entités collectives et les rapports sociaux. Les associations seront donc clairement posées comme le fruit de l'activité des individus, dans un rapport critique entre le but objectivable et l'intention. Comme l'écrit P. Raynaud, "Chez Weber, les entités collectives apparaissent à nouveau comme de simples données que la compréhension doit chercher à ramener à l'activité des individus : la notion d'activité sociale montre que les rapports sociaux euxmêmes ne peuvent être compris qu'à partir du point de vue d'un acteur individuel idéal ${ }^{48}$. Finalement, chez Weber, l'activité communautaire rejoint le comportement d'autrui, mais elle est d'abord activité rationnelle d'un individu posé idéalement. Certes, il existe bien un rapport entre les finalités réalisables à travers l'association et les fins personnelles; mais il est beaucoup moins formalisé que dans une philosophie morale. Un tel rapport n'est envisagé ici qu'en tant qu'explicatif des phénomènes sociaux : "La sociologie cherche donc à expliquer les phénomènes sociaux en comprenant les activités individuelles, c'est-à-dire en reconstituant le sens visé et les buts poursuivis par les acteurs ", écrit P. Raynaud ${ }^{49}$.

\subsection{De la coopération à l'association.}

Les associations et toutes les structures de la même société seront toujours ramenées chez Weber à des formes données de coopération. On comprend alors l'association, de quelque nature et de quelque taille qu'elle soit, comme structure conséquente à des activités de coopération. Apparemment, une telle approche rejoindrait celle d'Aristote, dans l'Éthique à Nicomaque. Cependant, chez Weber, l'association n'est que la résultante d'une activité spécifiquement humaine, (qu'il s'agisse de l'État, ou d'une association intermédiaire). Elle n'est jamais considérée dans son rapports au bien de la cité ou aux fins personnelles. Elle n'est pas comme tel objet d'analyse, mais elle permet de remonter à l'activité individuelle qui lui a donné naissance, et qui, seule, est " compréhensible ». Pour Weber dans "Essai sur quelques catégories de la sociologie compréhensive ", " des concepts comme ceux d'“État”, d'“association", de "féodalité" ou autres semblables désignent, d'une façon générale, du point de vue de la sociologie, des catégories représentant des formes données de la coopération humaine ; sa tâche consiste à les réduire à une activité

48. Raynaud, Philippe, Max Weber et les dilemmes de la raison moderne, p. 121. 49. Raynaud, Philippe, Max Weber et les dilemmes de la raison moderne, p. 101. 
“compréhensible", ce qui veut dire sans exception commune à l'activité des individus qui y participent ${ }^{50}$. Il va sans dire qu'une telle méthode s'oppose à l'emploi de l'analogie biologique, et à la conception de la société comme organisme préexistant. Pour Weber, l'unité fonctionnelle des phénomènes sociaux n'explique pas le «fonctionnement » social des individus. Weber a une recherche de causalité dans l'ordre de l'agir social, que me permet plus une union de la société comme super-individu. La vie sociale unifiée suppose a priori un fonctionnement de coopération. Si celui-ci n'est plus réalisé, on parlera de pathologie, mais sans trouver forcément la cause de dysfonctionnement. Les remarques de Weber sont pertinentes : en effet, la réalisation pratique de l'interdépendance entre des éléments d'un organisme vivant va de soi, lorsque l'unité de vie est réalisée à son niveau. Mais la réalisation d'une coopération effective dans l'ordre humain ne va pas de soi : elle présuppose la liberté individuelle. L'organisme sociétal, au sens de la tradition sociologique de Comte à Durkheim, est un modèle et peut être une conséquence, mais non pas une réalité immédiate; la structure sociale peut exister comme un champ général de l'action, mais pas l'organisme, du moins pas a priori.

Weber écrit, dans Économie et Société : "En vérité, ce n'est qu’à ce moment-là (avec l'interprétation compréhensive) que commence le travail de la sociologie (telle que nous l'entendons ici). En effet, dans le cas des "structures sociales" (à l'opposé des “organismes”), nous sommes en mesure d'apporter par delà la constatation de relations et de règles (les "lois" fonctionnelles) quelque chose de plus qui est éternellement inaccessible à toute "science de la nature" (au sens où elle établit les règles causales de processus et de structures et "explique" à partir de là les phénomènes singuliers) : il s'agit de la compréhension du comportement des individus singuliers qui y participent, alors que nous ne pouvons pas comprendre le comportement des cellules par exemple, mais l'appréhender seulement fonctionnellement et le déterminer ensuite d'après les règles de son développement ${ }^{51}$.

Comme nous pouvions le prévoir en fonction de la notion d'accord rationnel par finalité, "l'association à but déterminé » au sens de Weber est " une activité sociétaire impliquant une réglementation du contenu et des moyens de l'activité sociétaire, par accord rationnel par finalité, de tous les participants " 52 . Il faut noter que, chez Weber, l'analogie de « l'organe » est utilisée pour désigner les personnes chargées de l'exécution ( organes de l'association ${ }^{53}$ et "supports d'une activité sociétaire spécifique » ${ }^{54}$ ). Le concept d'association ainsi défini est relatif à "l'activité de socialisation ",

50. Weber, Max, 1913, Essai sur quelques catégories de la sociologie compréhensive, p. 319.

51. Weber, Max, 1921, Économie et société, collection "Recherches en sciences sociales ", Paris, Plon, 1971, pp. 13-14.

52. Weber, Max, Essai sur quelques catégories de la sociologie compréhensive, p. 329.

53. Weber, Max, Ibid., p. 329.

54. Weber, Max, Ibid., p. 320. 
" activité qui, d'après le sens dont on admet qu'il est visé subjectivement en moyenne, possède la signification d'un accord $»^{55}$ : Weber la distingue de l'activité sociétaire simplement orientée d'après cet accord. Il insiste donc sur l'accord entre les membres de l'association, accord qui est exprimé à travers les actes des membres associés. Exprimer l'accord davantage que l'unité semble signifier que Weber recherche un critère qui montre la spécificité humaine de l'association au plan de l'harmonisation des activités.

\subsection{Des diverses formes d'association.}

Par ailleurs, à l'intérieur du concept d'association, l'association à but déterminé (en tant qu'elle implique un accord et l'existence d'organes propres) est une structure sociale durable, constituée comme telle par un règlement. Mais il existe aussi des formes de socialisation occasionnelle que Weber définit par le concept d'échange. L'intérêt de ces deux pôles consiste dans la saisie par Weber d'une dynamique sociale conduisant de relations occasionnelle à la structure communautaire la plus durable. Il écrit («Essais sur quelques catégories de la sociologie compréhensive ») :

L'organe typique de la socialisation que nous appelons de nos jours «État » réside d'une part, dans de libres socialisations occasionnelles de brigands avides de rapines qui organisaient des raids sous la conduite d'un chef élu et d'autre part, dans la socialisation occasionnelle de la défense de ceux qui étaient menacés. La notion d'un " patrimoine » propre à l'association ainsi que la durée faisait entièrement défaut. Après le succès (ou l'échec) de l'expédition des pillards ou respectivement de la riposte des défenseurs, la socialisation se dissolvait. Le chemin est long et jalonné de transitions continues qui mena jusqu'à la socialisation durable de la caste militaire (...) et par delà, jusqu'à l'usurpation d'une activité sociétaire d'ordre juridique et administratif ${ }^{56}$.

Weber semble avoir été inspiré à ce niveau par Tönnies, pour qui " l'ère de la société suit celle de la communauté ${ }^{57}$. On doit reconnaître que, malgré le point de vue critique et conceptuel de Max Weber, il accorde une place importante à la dynamique des processus conduisant aux groupements humains (ou les dégradant), mais l'activité vitale (bien qu'impliquant une dynamique) est toujours construite sur la rationalité et l'individualité, jamais sur la communauté.

L'essai intitulé La Ville est un extrait de Économie et Société $(1921)^{58}$. Dans cet essai, Weber donne beaucoup d'applications concrètes de sa conception de l'association. La naissance de la ville, sous l'antiquité et au Moyen Âge, à partir ou non de diverses associations est examinée et comparée, pour

55. Weber, Max, Ibid., p. 330.

56. Weber, Max, Ibid., p. 355.

57. Tônnies, Ferdinand, 1887, Communauté et société, Paris, Retz, 1977, p. 80.

58. Weber, Max, La ville, Paris, Aubier, 1982. 
l'Orient et pour l'Occident. Au chapitre 1, traitant de l'organisation de la cité en Extrême-Orient, Weber écrit :

En Chine comme aux Indes, les guildes ou autres associations professionnelles disposaient de compétences définies, et les fonctionnaires se devaient d'en tenir compte. Il arrivait que les comités directeurs de ces associations usent d'importants pouvoirs de contrainte à l'encontre de tiers. Cependant, tous ces droits ou pouvoirs ne concernaient que quelques associations déterminées, et portaient sur quelques questions précises touchant leurs intérêts concrets. Toutefois, il n'y a pas, normalement d'organisation collective représentant la communauté des citadins en tant que tels. La notion même d'une telle possibilité est totalement absente. Ce qui manque avant tout, ce sont les attributs spécifiques du statut du $\operatorname{citadin}^{59}$.

En d'autres termes, Weber analyse l'existence des associations en Extrême-Orient, et remarque que ces associations, pour puissantes qu'elles soient parfois, ne sont jamais étendues à la communauté des citadins. On est donc renvoyé de la constitution des associations aux citadins : on constate qu'ils n'ont jamais formé une organisation collective et représentative, parce qu'on ne sait pas distinguer le « citadin » du «non citadin ». L'inexistence d'une structure associative renvoie donc, en sociologie compréhensive, à l'inexistence d'un accord et d'une volonté rationnelle entre des personnes voulant constituer une association. On comprend alors les exemples qui suivent :

Au Japon, l'organisation sociale était essentiellement féodale : les officiers ministériels faisaient face aux paysans, aux artisans, et aux commerçants, partiellement unis dans des associations professionnelles... La structure de la société indienne, faites de castes héréditaires séparant rituellement les professions les unes des autres, exclut tant la naissance d'une «bourgeoisie » que celle d'une " communauté urbaine ». Bien qu'il y eut plusieurs castes de marchands et de très nombreuses castes d'artisans ainsi qu'une masse d'intercastes, elles ne peuvent être considérées ensemble, ni traitées comme l'équivalent de l'ordre des bourgeois, tel qu'il existait en Occident $^{60}$.

L'existence d'un pouvoir centralisateur (Chine, Japon), ou de différences d'ordre culturel ou religieux, semble donc empêcher l'émergence des associations. Par rapport à ces cas de figure, Weber va analyser « la cité antique » et la « ville corporative d'Occident ».

La Cité antique était officiellement fondée sur une organisation de familles et, au dessus d'elles, sur des communautés qui reposaient souvent (au moins fictivement) sur la communauté d'ascendance et formaient des associations culturelles strictement exclusives par rapport à l'extérieur. Il est tout à fait significatif que, dans l'esprit de leurs membres, les cités antiques étaient des sociations librement consenties, et des confédérations d'associations de person-

59. Weber, Max, Ibid., p. 40.

60. Weber, Max, Ibid., p. 41. 
nes, qui avaient, pour partie, un caractère consanguin, et pour partie, comme dans le cas des fratries, en caractère militaire ${ }^{61}$.

Il est révélateur que Weber emploie ici le terme de sociation, qui met l'accent sur la constitution d'un groupe social homogène, d'un point de vue dynamique. Mais, il apparaît aussi que ces associations sont très partielles : elles reposent sur la lignée, la fratrie, etc. Une bonne partie des " citadins » en sont exclus : L'extension de la citoyenneté romaine à tout homme libre de l'empire ne date que de 212. Au contraire, la ville médiévale s'enracine dans l'expansion du christianisme, donc dans un idéal de fraternité. Aux V $\mathrm{V}^{\mathrm{e}}-\mathrm{VI}^{\mathrm{e}}$ siècles, les villes qui restent des foyers de civilisation se resserrent autour de l'évêque et de la vie chrétienne. Il est fondamental de comprendre (ce que Weber met remarquablement bien en lumière) que la ville médiévale s'origine dans la communauté chrétienne, " association confessionnelle d'individus, et non association rituelle de familles ${ }^{62}$. On passe ainsi d'associations particulières ayant droit de cité à une association plus universelle, parce que basée sur une foi et un engagement à vocation d'universalité. Ainsi, au moyen âge, selon Weber, c'est la "valeur exemplaire » de la communauté religieuse qui va fonder la fraternisation des citoyens.

L'irruption de la démocratie urbaine en Occident, datée de la fin du XI siècle, est caractérisée en Italie par le rôle des consuls. Weber écrit à ce propos :

Au début, ils semblent avoir été recrutés le plus souvent dans le corps des officiers nobles et parmi les chevaliers de la curie épiscopale ou seigneuriale ; c'est seulement ensuite que leur élections par la bourgeoisie conjurée, ou par ses représentants, se substitua à la nomination par les seigneurs de la ville... Bientôt, le mouvement d'opposition à l'organisation féodale l'emporta. Il fut interdit aux consuls de recevoir un fief de la part d'un seigneur ou d'exercer le commandement en tant que vassal ${ }^{63}$.

Notons au passage que, selon la méthode de Weber, c'est la qualité ou la fonction des individus qui explique cette forme d'organisation qui devint représentative et associative. Après l'énumération des privilèges acquis par les bourgeois, l'auteur resitue la genèse de cette nouvelle organisation :

Ainsi, à partir d'une simple groupement de personnes liées par serment, formé suivant les circonstances et à court terme, naquit un groupement politique durable dont les membres légalement associés jouissaient du droit statutaire particulier de citoyen d'une ville... Le droit de cité était un droit statutaire conféré au membre adhérent de l'association civique en tant que communauté liée par un serment ${ }^{64}$.

61. Weber, Max, Ibid., p. 57.

62. Weber, Max, Ibid., p. 63.

63. Weber, Max, Ibid., p. 72.

64. Weber, Max, Ibid., p. 73. 


\section{$374 \cdot$ Philosophiques / Automne 2000}

Outre l'association des bourgeois constitutive de la ville médiévale, il faut évoquer les groupements professionnels ou corporations. Freund (1982) écrit dans la préface :

Il arrive à Weber de rectifier une idée courante, précisément à propos des corporations. Le mouvement corporatif des artisans est apparu assez tard dans la lutte pour l'autonomie des villes. Initialement, dit-il, les révolutions étaient presque partout menées par des corporations qui n'étaient pas des corporations d'artisans. Partout où il l'emporta, il est vrai, le système corporatif a coïncidé avec l'apogée des villes tant dans leurs relations avec l'extérieur, que du point de vue de leur autonomie interne. C'est d'ailleurs uniquement dans ce cadre qu'on peut parler de démocratie médiévale ${ }^{65}$.

Au plan des groupements professionnels, c'est encore la fraternité qui crée un fondement de l'union, union liée à une reconnaissance religieuse. «De nombreuses associations professionnelles prirent soin de se donner pour premières obligations celles qui relevaient de sociabilité et de la religion ; elles prirent donc soin de chercher la reconnaissance religieuse, comme l'ont fait la plupart des guildes, et en pratique, toutes les corporations médiévales ${ }^{66}$.

Les corporations sont donc nées après l'association des citoyens, dans un climat permettant des unions au niveau des diverses professions. La remarque est d'importance, car Max Weber semblerait rappeler que l'association volontaire des citoyens en une communauté urbaine a été primordiale pour permettre des groupements de portée plus limitée. C'est la corporation des citoyens qui conditionne la naissance des corporations d'artisans, de commerçants : en d'autres termes, l'association comme forme de coopération politique doit constituer un pouvoir démocratique pour permettre d'existence des autres formes d'association. Le politique (ici la réalisation de la démocratie urbaine) en tant qu'engagement de toute la dimension communautaire des individus semble créer un milieu de vie qui, à son tour, va susciter diverses communautés intermédiaires qui correspondront aux dimensions professionnelles, religieuses, etc., des citoyens de la ville. Cette réflexion semble utilisable dans le monde socio-politique d'aujourd'hui : la responsabilité des citoyens à l'égard de leurs villes et de ceux qui y vivent passe par l'engagement des associations de quartiers, en co-responsabilité avec les municipalités. Les associations à objets plus limités, ou les organisations professionnelles ne suffisent pas pour faire vivre la ville, car un engagement de citoyen y parait aujourd'hui nécessaire.

\section{Conclusion.}

Il semble opportun au terme de ce travail, de réunir les éléments issus de divers champs du savoir et de diverses méthodes mises en jeu, et qui constituent

65. Freund, Julien, Préface de La ville (Max Weber), pp. 7-16, Paris, Aubier, 1982, p. 14. 66. Weber, Max, La ville, p. 78. 
l'intelligibilité du concept d'association chez Émile Durkheim, et chez Max Weber. En se basant entre autres sur les travaux du zoologiste Edmond Perrier, Durkheim cherche à replacer la société dans une tendance évolutive vers une individualité nouvelle. Cet aspect évolutif de l'analogie biologique le pousse à voir l'association comme fruit d'un processus d'intégration (physiologique) des individualités successives. Un autre aspect de l'analogie biologique aboutit à assigner à l'État dans la société, le même rôle qu'au cerveau dans l'organisme. Ces deux conclusions sont loin d'être purement idéologiques, elles se basent sur de multiples faits sociaux constatés dans les sociétés les plus diverses. Mais l'évolution constatée à travers l'histoire de France jusqu'à l'époque de Durkheim allait simultanément dans le sens d'un renforcement de la centralisation de l'État et du développement de certaines associations (syndicats, sociétés de secours mutuels, etc.). C'est pour cela que Durkheim donne à l'association, d'une part, le sens de réalité organique au sommet de laquelle culmine l'État, d'autre part le sens de groupe secondaire dynamisant la société et permettant l'expansion de l'individu. La signification du concept d'association chez Émile Durkheim intègre dans une large mesure une démarche expérimentale et les conclusions de la science des faits sociaux, l'analogie entre le vivant et la société à travers la science de son temps, mais aussi des éléments hérités de la philosophie politique. En effet, les concepts de coopération et d'association renvoient à une expériences, à des structures et à des réalités humaines, ils en sont pas nés de rien. Ils renvoient aussi à la lecture des philosophes qui ont précédé l'auteur, comme Montesquieu, Tocqueville et Renouvier. La découverte qu'ont fait ces auteurs au sujet de l'association comme corps intermédiaire entre l'individu et l'État a certainement influencé la synthèse corporative de Durkheim, même si celle-ci semble laisser à l'État le rôle primordial. L'ordre corporatif proposé par Durkheim est proprement politique, mais n'évite pas l'ambiguïté, car la conjonction des deux types d'association (corps intermédiaires et État) laisse flotter un doute sur la localisation du véritable centre de décision.

Le point de départ de Weber est tout autre : c'est l'individu en tant que sujet capable d'activités morales et de coopération. Ainsi, la vie n'est pas spécifiquement sociale, hypostasiée dans un super-organisme; elle est propre à l'individu, et peut s'exprimer à travers des activités intellectuelles, morales, sociales diverses et qui s'orientent vers des fins choisies avec plus ou moins de liberté. Ceci est aussi un point de départ éminemment philosophique et totalement différent. Au lieu de découvrir une analogie (ou une transposition ?) pour signifier l'être social supposé, on cherche à découvrir la personne humaine comme réalité existante et s'exprimant à travers des dimensions analogues, puisqu'impliquant toutes des "activités subjectivement significatives ». Nous pensons personnellement que cette approche de Weber est plus réaliste à l'égard de l'implication de la personne humaine visà-vis des autres et de la société. Cette même forme de réalisme conduit Weber à concevoir la première expression de la dimension politique de l'homme à 
travers l'activité de coopération. C'est la coopération dans ses diverses formes qui fonde les associations et les différentes structures sociales. Toutefois, cette coopération n'est pas envisagée en rapport avec une quelconque fin sociétale : nous ne sommes pas en philosophie politique. La démarche consiste à établir un lien entre les structures et le comportement des individus dans l'association (leur accord, leurs actes, le type de socialisation qui leur est propre). Weber analyse donc concrètement à travers le déroulement de l'histoire, les structures communautaires, leurs compositions, leurs rites, la manière de s'y engager et d'y prendre les décisions. Il s'intéresse beaucoup aux corporations auxquelles il attribue un réel statut de corps intermédiaires. Nous sommes ici en présence d'une approche sociologique de l'association (ou plutôt des associations) qui intègre un certain regard philosophique sur l'expérience de coopération sans présupposer formellement toute l'analyse de la philosophie morale et politique. 